\title{
Elastobuoyant Heavy Spheres: A Unique Way to Study Nonlinear Elasticity
}

\author{
Aditi Chakrabarti and Manoj K. Chaudhury \\ Department of Chemical and Biomolecular Engineering, Lehigh University, \\ Bethlehem, Pennsylvania 18015, USA
}

Serge Mora*

Laboratoire de Mécanique et de Génie Civil, UMR 5508, Université de Montpellier and CNRS, 163 Rue Auguste Broussonnet, F-34090 Montpellier, France

\section{Yves Pomeau}

Department of Mathematics, University of Arizona, Tucson, Arizona 85721, USA

(Received 8 May 2016; revised manuscript received 7 September 2016; published 28 December 2016)

\begin{abstract}
Large deformations in soft elastic materials are ubiquitous, yet systematic studies and methods to understand the mechanics of such huge strains are lacking. Here, we investigate this complex problem systematically with a simple experiment: by introducing a heavy bead of radius $a$ in an incompressible soft elastic medium. We find a scaling law for the penetration depth $(\delta)$ of the bead inside the softest gels as $\delta \sim a^{3 / 2}$, which is vindicated by an original asymptotic analytic model developed in this article. This model demonstrates that the observed relationship is precisely at the demarcating boundary of what would be required for the field variables to either diverge or converge. This correspondence between a unique mathematical prediction and the experimental observation ushers in new insights into the behavior of the deformations of strongly nonlinear materials.
\end{abstract}

DOI: 10.1103/PhysRevX.6.041066

Subject Areas: Materials Science, Soft Matter

\section{INTRODUCTION}

Singularities are pervasive in various problems of linear continuum mechanics. In wetting phenomena, stress diverges at a moving contact line [1,2]; it diverges at the tip of a crack or even at a sharp point indenting a plane [3]. Understanding how such singularities can be tempered has often given rise to new physics invariably prompting us, on many occasions, to investigate a material phenomenon at a molecular dimension and then herald a way to bridge the near field with the far-field behavior in a rather nontrivial manner. Nature, however, performs the difficult task and leaves its signature in a way that is independent of the constitutive property of a material, yet it belongs to a class of universality. Here, we report such a universality, which is discovered in the large deformation behavior of ultrasoft gels. Soft solids undergoing huge deformations exhibit various fascinating and puzzling mechanical behaviors [4-15]. Our experimental protocol to study extra-large elastic deformations is remarkably simple, in that a heavy bead of stainless steel is gently deposited on the horizontal

*serge.mora@umontpellier.fr

Published by the American Physical Society under the terms of the Creative Commons Attribution 4.0 International license. Further distribution of this work must maintain attribution to the author(s) and the published article's title, journal citation, and DOI. flat surface of a gel. The compliant gel is deformed by the load exerted by the heavy bead. It reaches a stable (elastobuoyant) equilibrium position when the elastic force exerted by the surrounding gel balances its weight [14], within a few tenths of a second. This experiment can be viewed as an elastic analog of the falling-ball viscometry, in which the bead reaches a terminal sedimentation velocity resulting from the balance of the bead's weight and the viscous drag force [16]. In the limit of Hookean elasticity, an analogy with the Stokes equation (by replacing shear viscosity with shear modulus and velocity with depth of submersion, $\delta$ ) [17] suggests that $\delta \sim a^{2}, a$ being the sphere radius. While for the higher elastic moduli gels [Fig. 1(a)], such a relationship is more or less valid, for the softer ones, when the bead is totally engulfed by the gel and the deformations are very large [Fig. 1(b)], it is observed that the depth scales with the bead's radius raised to an exponent of $3 / 2$. This is a nontrivial result that cannot be explained by the usual neo-Hookean model, i.e., by considering a quadratic elastic energy density with respect to finite strains. According to this model, the displacement would be infinite. When the same problem is analyzed using the original analysis presented in this paper, it is found, remarkably, that the exponent of the radius of the bead is just at the juncture of what would be required for the field variables to avoid divergence. What is quite remarkable about this analysis is that we do not need to speculate about any detailed nonlinear behavior of elastic solids 
[18-22]. The scaling laws are universal, being independent of the particular constitutive law of the elastic material. Other examples of the absence of a crucial parameter in some important physical relation is the case of Newton's drag force at large Reynolds number: The drag is proportional to the square of the velocity but is independent of the viscosity, even though viscosity is crucial for dissipating energy. Similarly, in our system, the stress-strain relation at large strains is crucial for determining the field of deformation but, if the material is stiff enough at large strains, the details of this strong nonlinear limit of the elastic response disappear in the scaling law for delta as a function of the physical parameters. This unique correspondence between a mathematical prediction and the experimental results, thereby unfolding new physics of highly nonlinear deformations, is the subject of this paper.

\section{EXPERIMENTAL EVIDENCE OF ELASTOBUOYANCY}

Cross-linked polyacrylamide gels were used in the experiments reported below. The gel solutions were prepared according to the recipe published previously $[14,23]$ and cured in home-built glass containers $(70 \mathrm{~mm} \times 50 \mathrm{~mm} \times 40 \mathrm{~mm})$. The inner walls of the containers were grafted with a thin layer (about $5 \mathrm{~nm}$ ) of polydimethyl siloxane chains so that the gel solution contacted the walls at $90^{\circ}$ to ensure that the surface of the cured gel was flat. All the experiments were performed after two hours of gelation. For the estimation of the shear modulus of the gel, a linear elastic model was used in order to ensure consistency of quantification for all the gels. The shear modulus was determined from the resonant mode of vibration of a gel slab confined between two parallel glass slides [23]. Steel spheres (density $7.8 \mathrm{~g} / \mathrm{cc}$, diameters $1-10 \mathrm{~mm}$ ) were gently placed one by one on the gel surface, and its side-view image was captured by a camera.
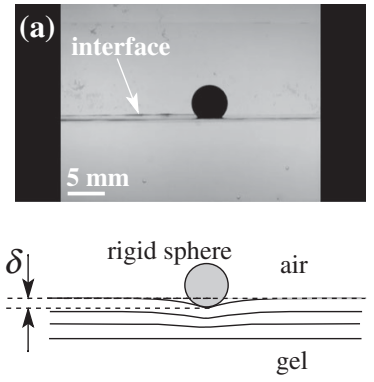

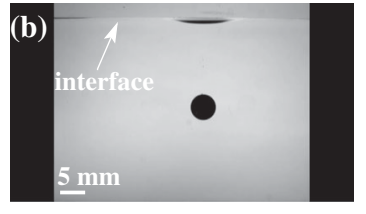

air

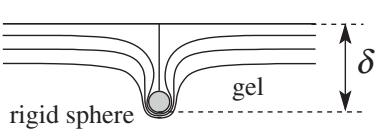

FIG. 1. Snapshots and schematics of the experiment. (Top panels) Side views of two transparent cells filled with a polyacrylamide gel with shear modulus $1160 \mathrm{~Pa}$ (a) and $13 \mathrm{~Pa}$ (b). Two identical steel beads ( $5 \mathrm{~mm}$ diameter) have been placed on the free air-gel interface. The vertical downshifts are, respectively, $\delta=0.03 \delta_{0}$ and $\delta=320 \delta_{0}$. (Bottom panels) Schematic representation of the deformation fields in the respective experiments.
Dissipative processes within the gel dampened any oscillations, and the spheres sank until they became stagnant in the polyacrylamide gel, the whole process taking only fractions of a second. The depth of submersion, $\delta$, was measured from the upper surface of the gel until the base of the sphere, which denoted the net downward displacement due to the inclusion of the spherical particle by the surface (Fig. 1). The cells are large enough to avoid any finite boundary effects, from the side walls as well as from the bottom. The measurement of depth for each sphere was made in the central region of the container. After each measurement, the sphere was gently removed from the gel using a magnet, held slightly away from the free surface. We waited for a few minutes between each measurement, which ensured there was no memory of the position of the previous sphere inside the gel.

If the bead is too small and the gel is stiff $[7,24]$, the surface bends slightly under the weight of the bead and $\delta \ll a$ [Fig. 1(a)]. By increasing the bead radius or decreasing the elastic modulus, the particle submerges itself to a considerable depth inside the gel. The surface of the gel wraps around the particle and closes to create a line singularity connecting the particle to the free surface of the gel [Fig. 1(b)]. Strings of tiny air bubbles appear in this thin channel, which soon coalesce and escape through it while the channel further closes because of the auto-wetting forces of the gel's surface. If the surface of the gel is premarked with ink spots, it is easy to visualize that the surface of the gel becomes appreciably stretched while the sphere sinks through the gel, still connected to the free surface via a thin channel. These basic experiments were reported in a previous article [14] but without a detailed analysis. Here, we report a detailed set of experiments, in which the shear modulus of the gel was varied from $13 \mathrm{~Pa}$ to about $3000 \mathrm{~Pa}$.

Prior to subjecting these experimental results to a comparison with a theoretical analysis, we need to verify how meaningful it is to consider only the effect of elasticity by ignoring the surface tension of the gel in predicting the depth of submersion of the bead and how reversible the deformation of the gel is within the experimental time scale, i.e., a few seconds. The first question is partly philosophical; it rests upon the distinction between surface free energy $\gamma$ and surface tension. The latter differs from the former by surface stress $d \gamma / d e, e$ being the surface strain. As the major constituent of these amorphous gels is water, we expect that the surface stress is negligible. In fact, several recent studies that measured surface tensions of various amorphous soft polymers strongly suggest that their surface tensions are practically the same as their surface free energies [25]. Thus, we only need to figure out if the surface free energies of the gels play any role. This could be more or less resolved by comparing them with the equivalent spring constant of the sample. The latter can be obtained by slightly raising the height of the bead by an 
electromagnet and releasing it so that the bead undergoes an underdamped oscillation and reaches the neutral position. For three gels, in which the beads are completely submerged, the equivalent spring constants are estimated from the frequency of oscillation to be $0.2 \mathrm{~N} / \mathrm{m}, 5.6 \mathrm{~N} / \mathrm{m}$, and $13 \mathrm{~N} / \mathrm{m}$, and they increase systematically with their shear moduli (13 Pa, $140 \mathrm{~Pa}$, and $360 \mathrm{~Pa}$ ). Figure 2(a) shows a typical profile of such an oscillation. Comparing these spring constants with the surface tension $(0.07 \mathrm{~N} / \mathrm{m})$ of water, we conclude that the contribution of surface tension can be safely neglected for all the gels used in this study except, perhaps, for the lowest modulus gel for which the spring constant is 3 times that of the gel's surface tension. However, when a bead is completely submerged in the gel, any variation of the height of the sphere does not alter the area or the excess energy of the free surface of the gel. We thus believe that the surface tension spring does not play a significant role in determining the depth of submersion of the sphere as long as it is completely engulfed by the gel. Further support to this viewpoint, i.e., the dominant role of elasticity over surface tension, is gathered from the experiments described below, which also exemplify the reversibility of the deformations.

With respect to the second question, it is important for us to emphasize what type of reversibility of the gel is pertinent to the current studies, as a system loaded externally can deform with an appreciable dissipation of energy due to viscous or plastic flow. Fracture can also occur in the material if the load is large enough, or contrarily, it could get locked into a different state via internal rearrangement of bonds. The material would, therefore, not return to its original state when the load is removed. We performed certain experiments, as discussed below, which ensured that none of the above types of irreversibility is a concern within the time scale relevant for the elastobuoyancy experiments. The first experiment was performed by studying the variation of the depth of a steel bead $(2.8 \mathrm{~mm}$ diameter) inside a soft gel $(\mu=13 \mathrm{~Pa})$ by imposing an additional vertical magnetic force on the sphere. The depth of the bead was noted as a function of the increasing force in the loading cycle and similarly for the decreasing force in the unloading cycle [Fig. 2(b)]. These experiments were performed over a time scale of a few minutes. After repeating the experiments a few times, the sphere went back to its initial elastobuoyant position, showing no significant hysteresis (see Ref. [26] for a movie and more details). This kind of reversibility was also checked with a slightly stiffer gel $(\mu=101 \mathrm{~Pa})$ and a larger bead (10 mm diameter) (Ref. [26]). The above observations lead us to conclude that the deformation of the gel generated by a bead is predominantly reversible and controlled by elastic forces. This is the case even when the gel undergoes large deformations and, as will be shown below, when it is loaded with a sharp pointed needle.

The second experiment was performed by studying the sensitivity of the elastobuoyancy phenomenon to temperature-by increasing the system temperature, the elastic modulus of a polyacrylamide gel increases, and on cooling, such gels become softer with a corresponding decrease of the modulus. The depth of submersion of a bead inside the softest gel $(\mu \sim 13 \mathrm{~Pa})$ was studied by varying its stiffness in a temperature-controlled oven by covering its surface with a thin layer of paraffin oil to prevent the gel from drying out. The temperature of the gel was monitored by placing a thermometer inside an identical sample of gel in a similar-sized container, placed inside the oven. After the gel was heated to $70^{\circ} \mathrm{C}$, a 5-mm-diameter steel sphere was released into it through the layer of paraffin oil. The depth of the sphere was measured at this temperature while it was in the oven. As the gel was gradually cooled, the sphere sank deeper inside it. A waiting time of one hour before the measurement of the depth of the sphere at each
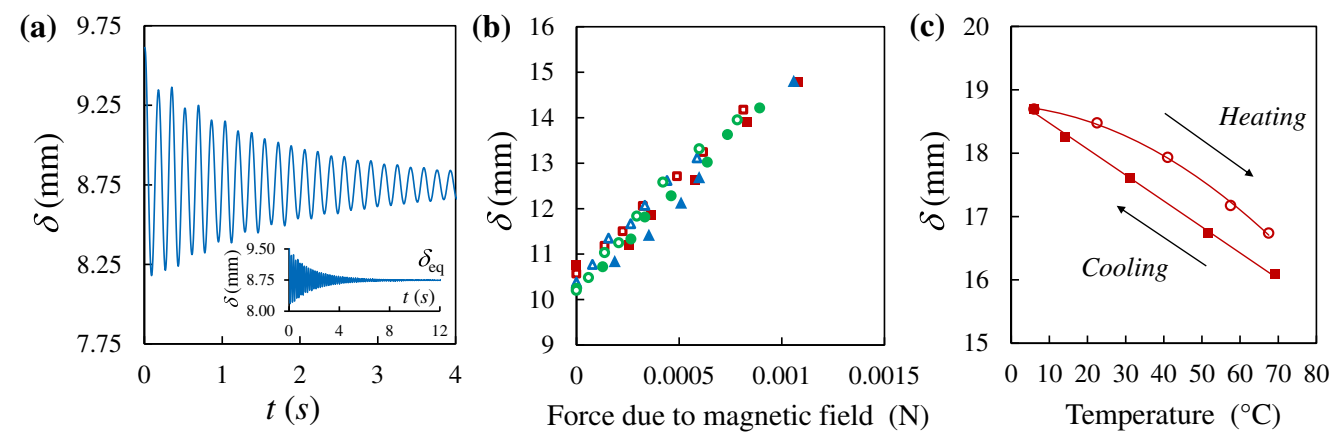

FIG. 2. Experimental evidence of reversibility of the gels. (a) A 10-mm-diameter steel sphere immersed in a 140-Pa gel, when slightly disturbed from its elastobuoyant position via an electromagnet, undergoes underdamped oscillations about its equilibrium depth $\delta_{\text {eq }}$. (b) Depth of an elastobuoyant bead (2.8 mm diameter) in a 13-Pa gel is plotted as a function of the strength of an external vertical magnetic force. The depth at zero force indicates the equilibrium-elastobuoyant position of the sphere. The data (red, blue, and green) shown here are from three different experiments, where the closed symbols indicate the loading cycles and the open symbols indicate the unloading cycles. (c) The depth of submersion $\delta$ of a 5-mm-diameter steel sphere in a soft gel $(\mu \sim 13 \mathrm{~Pa})$ is shown, varying as a function of its temperature. The experiments in the cooling cycle were performed first, after which the gel was heated systematically to obtain the data for the heating cycle. 
temperature allowed the gel to equilibrate reasonably well. After the gel was cooled to about $5{ }^{\circ} \mathrm{C}$, it was heated again, which decreased the depth of the embedded steel sphere [heating cycle, Fig. 2(c)]. The depth of submersion of the sphere plotted as a function of the temperature for both the cooling and the heating cycles [Fig. 2(c)] shows that there is a little hysteresis in this system, in that the difference in the depths of the sphere for a given temperature is within 5\%. Taken together, we conclude that the gels are thermomechanically stable, and the deformations of the gel generated by a bead are predominantly reversible and controlled by elastic forces.

The penetration depth $\delta$ is plotted versus the radius $a$ of the steel spheres in log scales in the upper inset of Fig. 3, for ten different gels ( $\mu$ : $13 \mathrm{~Pa}-2930 \mathrm{~Pa})$ and different radii. The upper white region of this inset shows the data points for beads that were completely below the surface of the gel. The nondimensionalization of the depths, as well as the radii of each bead in each gel by the intrinsic length scale $\delta_{0}$ defined as $\delta_{0}=(\mu / \Delta \rho g)$ (Fig. 3) (where $\mu$ is the shear modulus of the gel, $\Delta \rho$ is the apparent density of the buoyant spheres, and $g$ is the gravitational acceleration), shows that all the data cluster around a mean master curve with two distinctive asymptotic limits. The value of $\Delta \rho$ varied between $\left(\rho_{\text {steel }}-\rho_{\text {gel }}\right)$ for the fully engulfed beads to $\left(\simeq \rho_{\text {steel }}\right)$ for the beads that were almost on the surface of the stiffer gels. Thus, for each sphere-gel system, $\Delta \rho$ was precisely evaluated from experimental images on the basis of the volume of the bead engulfed by the gel, analogous to the Archimedes principle of buoyancy for flotation on a liquid [27]. The normalized data can be divided more or

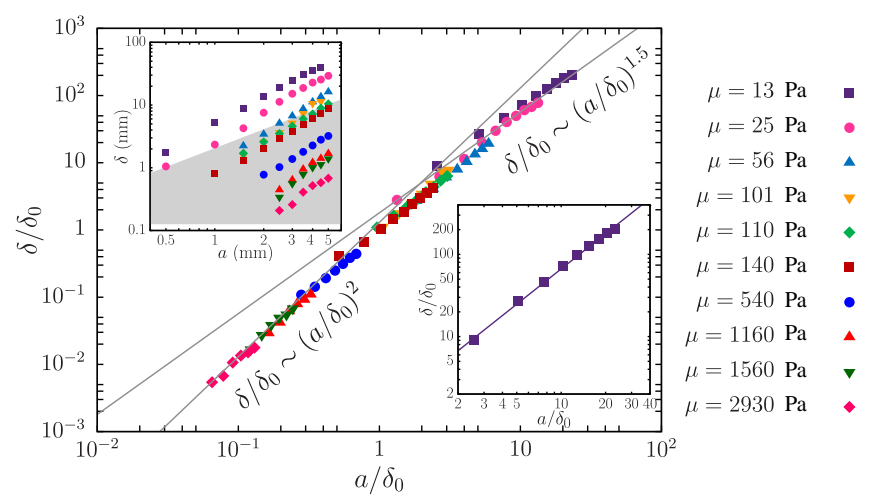

FIG. 3. Dimensionless depth of spheres $\left(\delta / \delta_{0}\right)$ plotted as a function of its dimensionless radius $a / \delta_{0}$, for various shear moduli of the gels from $\mu=13 \mathrm{~Pa}$ to $2930 \mathrm{~Pa}$. The grey lines indicate power-law curves $\left[\delta / \delta_{0} \sim\left(a / \delta_{0}\right)^{\alpha}\right]$ with $\alpha=2$ and $\alpha=1.5$ for the two asymptotic limits for the normalized data. (Upper inset) Depths $(\delta)$ versus radii $(a)$ for all the spheres. The plot area is divided into two domains, the boundary indicating $\delta=2 a$. The data points above the boundary indicate that the spheres were entirely below the gel's surface. (Lower inset) Best fit $\left[\delta / \delta_{0}=k\left(a / \delta_{0}\right)^{\alpha}\right]$ for the softest gel (13 Pa) highlighted (see text). less into two regimes: one with the nondimensional radii $a / \delta_{0}<1$ and the other with $a / \delta_{0}>1$, with an intermediate transition regime. A fit of the nondimensional depths as a function of the nondimensional radii for the regime $a / \delta_{0}<1$ with the power-law function $\left(\delta / \delta_{0}\right)=k\left(a / \delta_{0}\right)^{\alpha}$ with adjustable parameters $\alpha$ and $k$ yields $\alpha=1.96 \pm 0.06$ and $k=1.09 \pm 0.1$. The error bars are obtained from a 95\% confidence-limit analysis. We conclude that for $a / \delta_{0}<1$, the depths as a function of the radii follow $\delta \sim a^{2}$ within the error limits. On careful examination of the experimental points corresponding to large deformations, i.e., $a>\delta_{0}$, we find that the data corresponding to the two softest gels $(\mu: 13 \mathrm{~Pa}$ and $25 \mathrm{~Pa})$ are shifted from the rest of the data because their multiplicative factor $(k)$ is significantly larger than the rest of the data for the other gels. This indicates that the length scale $\delta_{0}$, which is defined with the elastic linear properties of the sample, is therefore not sufficient to describe the whole data accurately, and nonlinear effects have to be taken into account. We conclude that all the data in this regime $\left(a / \delta_{0}>1\right)$ cannot be investigated together. It is more appropriate to investigate the data for each gel composition separately, i.e., for a given nonlinear material stress-strain relationship. By fitting the data for the two softest gels $(\mu: 13 \mathrm{~Pa}$ and $25 \mathrm{~Pa})$, where all the beads are completely engulfed, with the power-law function, we find that $\alpha_{13 \mathrm{~Pa}}=1.42 \pm 0.05$ (see lower inset of Fig. 3), $k_{13 \mathrm{~Pa}}=2.52 \pm 0.3$ and $\alpha_{25} \mathrm{~Pa}=1.52 \pm 0.09$, $k_{25} \mathrm{~Pa}=1.63 \pm 0.3$. The exponents for the fits for the gels $\left(a / \delta_{0} \sim 1\right)$ in the intermediate regime lie between $\alpha \sim 1.5$ and $\alpha \sim 2$. Thus, from the experimental observations, we infer that in the limit of $a$ significantly greater than $\delta_{0}$, the general trend is close to $\delta \sim a^{p}$, where $p$ is in the range of $1.4-1.5$, within the error limits. Thus, we conclude that the power law observed $\left(\delta \sim a^{2}\right)$ in the gels of higher shear moduli is closer to the regime already studied before what one would expect in analogy with the elastic Stokes equation in the Hookean limit. What is astounding is the observation of an exponent close to $1.5\left(\delta \sim a^{1.5}\right)$ in the case of the gels where the beads are entirely below the surface of the gel; i.e., $\delta>2 a$. The small uncertainty of the exponent (less than 6\%) clearly indicates a different regime, as compared to the higher modulus gels.

Note that an exponent close to 1.5 for $a$ is also fully supported by the experimental scaling law that we found from indentation tests of the gels, performed with sharp needles as shown in Sec. IV.

In order to interpret these novel observations in the ultrasoft gels, we develop a new model to tackle such extra-large deformations in the following section.

\section{ASYMPTOTIC ANALYTIC MODEL FOR LARGE ELASTIC DEFORMATIONS}

From the outset, a motivating picture of the problem can be gleaned from the comparison of the potential energy of the bead in the gravitational field and the energy of the 
elastic deformation of the gel. In the limit of small deformations, the elastic deformation energy is given by the elastic modulus times the volume integral of the square of the strain. The strain is displacement $(\delta)$ divided by range of this displacement (radius of bead, $a$ ). Therefore, the elastic energy scales as $\mu a^{3}(\delta / a)^{2} \sim \mu \delta^{2} a$, which is to be compared with the gravitational potential energy $\Delta \rho g a^{3} \delta$. This leads to the scaling $\delta \sim a^{2}$. However, the experimentally observed scaling $\delta \sim a^{3 / 2}$ implies that the elastic energy in an extremely large deformation must scale as $\delta^{3}$. The detailed analysis based on a model, presented in this paper for the first time, shows that the above scaling is nontrivial. Moreover, the scaling result that follows is independent of the constitutive laws of an elastic material.

\section{A. Gravity energy of engulfed spheres}

Consider deformations that preserve the volume, as is the case with the elastic gels used in the experiments. Since the bead is supposed to be totally engulfed with $\delta \gg a$, the surface of the deformed gel is fairly flat and horizontal. A supplementary (virtual) downshift $\delta^{\prime}$ produces an opposite rise of the same volume of gel. Therefore, the gravitational energy variation of the system consisting of the sphere plus the gel is $\frac{4}{3} \pi a^{3} \Delta \rho g \delta^{\prime}$. We conclude that the gravitational energy shift is $\mathcal{E}_{\mathrm{gr}} \simeq \frac{4}{3} \pi a^{3} \Delta \rho g \delta$ in the limit we are considering.

\section{B. Outline of calculating the elastic energy in the limit $\delta \gg a$}

The axis of symmetry being the vertical axis, the two coordinates changed by the deformation are the radius in the horizontal plane, $r$, and the vertical coordinate $z$. The deformation maps the undisturbed state with coordinates $(r, z)$ to a disturbed state $[R(r, z), Z(r, z)]$, or, in radial coordinates, from coordinates $(\tilde{r} ; \theta)$ to $[R(\tilde{r}, \theta) ; Z(\tilde{r}, \theta)]$, with $\tilde{r}$ the radius and $\theta$ the polar angle of the $(r ; z)$ plane [see Fig. 4(d)]. The elastic energy is a function of the strain tensor (also called the right Cauchy-Green deformation tensor), which gives the square of local change in distances due to deformation: $C=F^{T} F$, with $F$ being the deformation gradient tensor [28]. In the absence of any preferred direction, the elastic energy density $W$ of an incompressible solid may depend only on two scalars (invariant under global rotation) that can be made out of the strain tensor: the trace $\left(I_{1}\right)$ and the sum of the square of its components $\left(I_{2}\right)$. In cylindrical coordinates with an azimuthal invariance, these invariants read $I_{1}=$ $R_{, r}^{2}+\left(R^{2} / r^{2}\right)+R_{, z}^{2}+Z_{, r}^{2}+Z_{, z}^{2}$ and $I_{2}=\left(R_{, r}^{2}+Z_{, r}^{2}\right)^{2}+$ $\left(R_{, z}^{2}+Z_{, z}^{2}\right)^{2}+2\left(R_{, r} R_{, z}+Z_{, z} Z_{, r}\right)^{2}+\left(R^{4} / r^{4}\right)$, where indices preceded by a comma denote partial derivatives. For the neo-Hookean model, which is commonly used to describe soft gels, the strain energy density is $W=(\mu / 2) I_{1}$. However, beyond a certain deformation, the neo-Hookean model cannot be a fair representation of

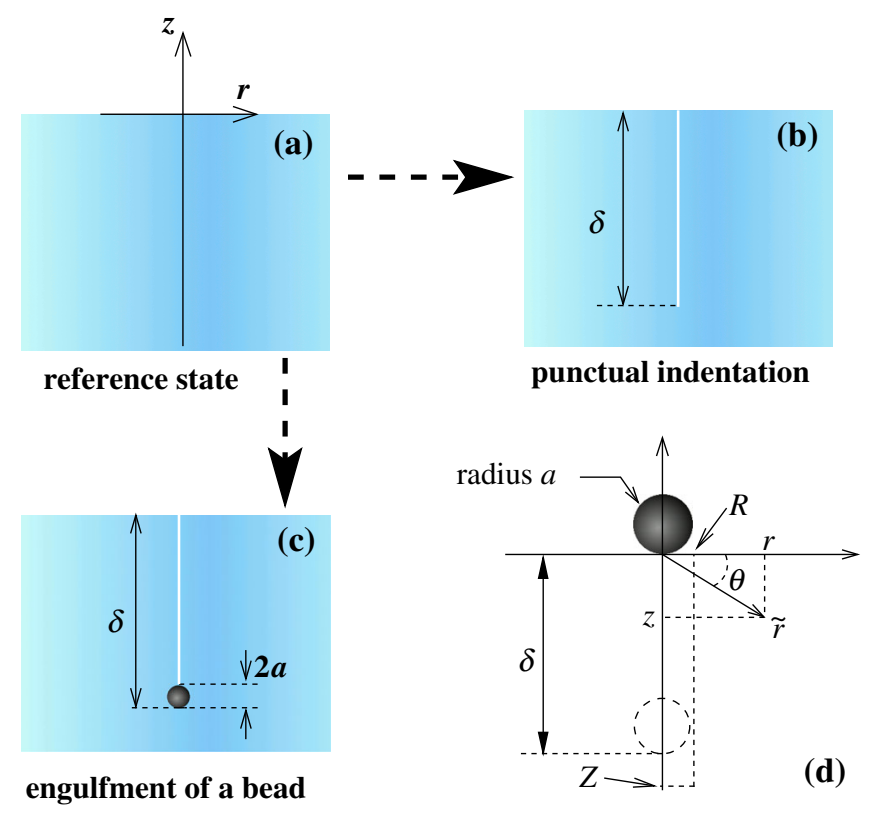

FIG. 4. Sketches corresponding to the steps of the calculation of the elastic energy. (a) Reference state (no deformation). (b) A point load applied at the free surface. The displacement at the application point is $\delta$. (c) A sphere of radius $a$ indents the free surface over the distance $\delta$. (d) Mapping from the reference state (solid lines, with the bead at the surface) to the deformed state. A point of the gel in the rest state is located with coordinates $(r, z)$. Note that $\tilde{r}$ is the distance from the initial contact point of the bead. In the deformed state, the point that was at $(r, z)$ is located at $R(r, z), Z(r, z)$.

rubberlike materials whose elasticity originates from unfolding of polymer chains: Once these chains reach their full extension, the energy cost for a supplementary unfolding diverges so that a further deformation is accompanied by a diverging additional elastic energy, in contrast with the ideal neo-Hookean law (see Fig. 6). In what follows, we do not restrict ourselves to the neoHookean case.

The deformation field of the gel can be described with two characteristic lengths - the downshift of the sphere $\delta$ and the radius of the sphere $a$. Here, we are dealing with the limit of large downshifts, that is, $\delta$ far larger than $a$. Below, we demonstrate that, in this asymptotic case, the elastic energy of the system does not depend on $a$ in the limit $a \ll \delta$. As a first step, we consider a normal point load applied to the free elastic surface at $(r, z)=(0,0)$ [Fig. 4(b)], and we find a scaling law for the elastic energy per unit volume at a given distance $\tilde{r}$ from the loading point. The condition for the elastic energy to be convergent is established. Then, as a second step, we consider a hard sphere with a finite radius, producing a displacement $\delta$ at the contact point $(r, z)=(0,0)$ equal to the displacement at $(r, z)=(0,0)$ induced by the previously considered pointlike load. We demonstrate that for $\delta \gg a$, the elastic energies associated with the displacement field generated 
by the sphere or the pointlike load are equal: These energies are independent of $a$ and proportional to $\delta^{3}$.

\section{Elastic energy for a pointlike load}

Since we are interested in the case $a \ll \delta$, we first deal with the limit of a pointlike force acting vertically on the free horizontal surface of an elastic solid, and we find scaling laws for the strain by first assuming a particular form for the strain-density elastic energy.

Incompressibility of the elastic medium is imposed by writing that the determinant $\mathcal{D}$ of the first derivatives of $\mathbf{R}(x, y, z)$ is equal to 1 . For axisymmetric deformations, $\mathcal{D}=(R / r)\left(R_{, r} Z_{, z}-R_{, z} Z_{, r}\right)$. The total energy of the system $\{$ gel + sphere $\}$ reads

$\mathcal{E}=\frac{4}{3} \pi \Delta \rho a^{3} g Z(0,0)+2 \pi \int_{-\infty}^{0} \mathrm{~d} z \int_{0}^{\infty} \mathrm{d} r(W-q \mathcal{D}) r$,

where the Lagrange multiplier $q(r, z)$ imposes the incompressibility condition $\mathcal{D}=1$ [29].

For the pointlike heavy sphere we first consider here [see Fig. 4(b)], the weight of the bead can be reduced to a point force located at $(r, z)=(0,0)$. Equation (1) simplifies to

$$
\begin{aligned}
\mathcal{E}= & \frac{16 \pi^{2}}{3} \Delta \rho a^{3} g \int_{-\infty}^{0} \mathrm{~d} z \int_{0}^{\infty} r Z(\mathbf{r}) \delta^{3}(\mathbf{r}) \mathrm{d} r \\
& +2 \pi \int_{-\infty}^{0} \mathrm{~d} z \int_{0}^{\infty} \mathrm{d} r(W-q \mathcal{D}) r
\end{aligned}
$$

where $\delta^{3}(r, z)$ is the 3D Dirac distribution.

The Euler-Lagrange conditions of minimization of the energy [Eq. (2)] read [5]

$$
\begin{aligned}
& \left(\frac{\partial(W-q \mathcal{D}) r}{\partial R_{, r}}\right)_{, r}+\left(\frac{\partial(W-q \mathcal{D}) r}{\partial R_{, z}}\right)_{, z}=\frac{\partial(W-q \mathcal{D}) r}{\partial R} \\
& \left(\frac{\partial(W-q \mathcal{D}) r}{\partial Z_{, r}}\right)_{, r}+\left(\frac{\partial(W-q \mathcal{D}) r}{\partial Z_{, z}}\right)_{, z}=\frac{8 \pi r \Delta \rho g a^{3}}{3} \delta^{3}(\mathbf{r})
\end{aligned}
$$

Let $\delta$ be the displacement of the gel at $(r ; z)=(0 ; 0)$. Note that $\delta$ is the unique relevant length for the displacement field. For points located at a distance to the load $\tilde{r}$ much smaller than $\delta$, the latter is no longer relevant at these short length scales. Therefore, no length scales are expected to occur in the scaling laws, and one assumes a power law of $\tilde{r}$ for the displacements for $\tilde{r} \ll \delta$ : $Z=$ $\delta+\tilde{r}^{\beta} f_{1}(\theta)$ and $R=\tilde{r}^{\gamma} f_{2}(\theta)$. Since the gel is vertically stretched and horizontally squeezed in the vicinity of the bead, one assumes that $\gamma>\beta$. This hypothesis will be checked later [see Eq. (16) below].

The constraint of conservation of volume reads (for $\tilde{r} \ll \delta)$

$$
\mathcal{D}=1 \Rightarrow \overbrace{\frac{R}{r}}^{\overbrace{}^{\tilde{r}}-1} \overbrace{R_{, r}}^{\sim \tilde{r}^{\gamma}-1} \overbrace{Z_{, z}}^{\sim_{\tilde{n}}^{\beta-1}}-\overbrace{R_{, z}}^{\sim \tilde{r}^{\gamma}-1} \overbrace{Z_{, r}}^{\sim_{\tilde{r}}^{\beta-1}})=1 \Rightarrow \gamma=\frac{3-\beta}{2} .
$$

The condition that $\gamma>\beta$ with Eq. (5) yields $\beta<1$.

We first assume that the strain energy density function $W$ is proportional to $I_{1}^{\alpha_{1}} I_{2}^{\alpha_{2}}$ (the exponents $\alpha_{1}$ and $\alpha_{2}$ are constant) in the range of strains undergone by the elastic solid in the vicinity of the application point, i.e., for $\tilde{r}$ far smaller than $\delta$. This choice for $W$ is crucial to obtain the scaling law, but it has no effect on the final results, as argued in Sec. III E: It does not limit the general nature of the theory.

The scaling laws for the first and second invariants of the Cauchy deformation tensor are

$I_{1}=\operatorname{Tr}(C)=\overbrace{R_{, r}^{2}}^{\sim \tilde{r}^{1-\beta}}+\overbrace{\frac{R^{2}}{r^{2}}}^{\tilde{r}^{1-\beta}}+\overbrace{R_{, z}^{2}}^{\sim \tilde{r}^{1-\beta}-\overbrace{Z_{, r}^{2}}^{\sim \tilde{r}^{2 \beta-2}}}+\overbrace{Z_{, z}^{2}}^{\tilde{r}^{2} \beta-2} \sim \tilde{r}^{2 \beta-2}$,

$$
\begin{aligned}
I_{2}= & \operatorname{Tr}\left(C^{2}\right) \\
= & \left(R_{, r}^{2}+Z_{, r}^{2}\right)^{2}+\left(R_{, z}^{2}+Z_{, z}^{2}\right)^{2}+2\left(R_{, r} R_{, z}+Z_{, z} Z_{, r}\right)^{2} \\
& +\frac{R^{4}}{r^{4}} \sim \tilde{r}^{4 \beta-4} .
\end{aligned}
$$

These scaling laws for $I_{1}$ and $I_{2}$ are used to obtain the scaling laws for the various terms of the CauchyPoisson equations [Eqs. (3) and (4)]. We first deal with Eq. (3):

$$
\begin{aligned}
& \frac{\partial(W-q \mathcal{D}) r}{\partial R_{, r}} \\
& =r \frac{\partial W}{\partial I_{1}} \frac{\partial I_{1}}{\partial R_{, r}}+r \frac{\partial W}{\partial I_{2}} \frac{\partial I_{2}}{\partial R_{, r}}-q r \frac{\partial \mathcal{D}}{\partial R_{, r}} \\
& =\quad \underbrace{2 r \frac{\partial W}{\partial I_{1}} R_{, r}}_{\sim \tilde{r}^{(3-\beta) / 2+2\left(\alpha_{1}+2 \alpha_{2}-1\right)(\beta-1)}} \\
& \quad+\underbrace{4 r \frac{\partial W}{\partial I_{2}}\left(R_{, r}\left(R_{, r}^{2}+Z_{, r}^{2}\right)+R_{, z}\left(R_{, r} R_{, z}+Z_{, z} Z_{, r}\right)\right.}_{\sim \tilde{r}^{(3-\beta) / 2+2\left(\alpha_{1}+2 \alpha_{2}-1\right)(\beta-1)}} \\
& \quad-q \underbrace{R Z_{, z}}_{\sim \tilde{r}^{(\beta+1) / 2}} .
\end{aligned}
$$


In the same way, one finds

$$
\begin{gathered}
\frac{\partial(W-q \mathcal{D}) r}{\partial R_{, z}} \sim \tilde{r}^{(3-\beta) / 2+2\left(\alpha_{1}+2 \alpha_{2}-1\right)(\beta-1)}, \\
\frac{\partial(W-q \mathcal{D}) r}{\partial R} \sim \tilde{r}^{(\beta-1) / 2} .
\end{gathered}
$$

From Eqs. (3) and (8)-(10), one obtains $\tilde{r}^{(1-\beta) / 2+2\left(\alpha_{1}+2 \alpha_{2}-1\right)(\beta-1)} \sim q \tilde{r}^{(\beta-1) / 2}$, and the scaling law for the Lagrange multiplier is

$$
q \sim \tilde{r}^{(\beta-1)\left(2 \alpha_{1}+4 \alpha_{2}-3\right)} .
$$

The last Cauchy-Poisson equation [Eq. (4)] is now used to get an expression of $\beta$ as a function of $\alpha_{1}$ and $\alpha_{2}$. It can be written

$$
\frac{1}{r}\left(r \frac{\partial(W-q \mathcal{D})}{\partial Z_{, r}}\right)_{, r}+\left(\frac{\partial(W-q \mathcal{D})}{\partial Z_{, z}}\right)_{, z}=\frac{8 \pi \Delta \rho g a^{3}}{3} \delta^{3}(\mathbf{r}) .
$$

Denoting the two quantities on the left-hand side of Eq. (12) as $\sigma_{z r}=\left(\partial(W-q \mathcal{D}) / \partial Z_{, r}\right)$ and $\sigma_{z z}=$ $\left(\partial(W-q \mathcal{D}) / \partial Z_{z}\right)$, one finds that this is the divergence (expressed in cylindrical coordinates) of the $z$ components of the stress tensor $\sigma$ [28].

We first calculate the scaling laws for $\sigma_{z r}$ and $\sigma_{z z}$ using Eqs. (5), (6), and (11):

$$
\begin{aligned}
\sigma_{z r} & =\frac{\partial(W-q \mathcal{D})}{\partial Z_{, r}} \\
& =\frac{\partial W}{\partial I_{1}} \frac{\partial I_{1}}{\partial Z_{, r}}+\frac{\partial W}{\partial I_{2}} \frac{\partial I_{2}}{\partial Z_{, r}}-q \frac{\partial \mathcal{D}}{\partial Z_{, r}} \\
& =\underbrace{2 \frac{\partial W}{\partial I_{1}} Z_{, r}}_{\sim \tilde{r}^{(\beta-1)\left(2 \alpha_{1}+4 \alpha_{2}-1\right)}}+\underbrace{q \frac{R}{r} R_{, z}}_{\sim \tilde{r}^{1-\beta}} .
\end{aligned}
$$

The second term of the right-hand side of Eq. (14) scales as $\tilde{r}^{(\beta-1)\left(2 \alpha_{1}+4 \alpha_{2}-3\right)} \tilde{r}^{1-\beta}$. It is negligible when $\tilde{r}$ tends to zero when compared to the first term of Eq. (14), provided that the exponent of the second term is larger than the exponent of the first one, i.e.,

$(\beta-1)\left(2 \alpha_{1}+4 \alpha_{2}-3\right)+1-\beta>(\beta-1)\left(2 \alpha_{1}+4 \alpha_{2}-1\right)$,

which is formally equivalent to $\beta<1$. Since it is assumed from the beginning that $\beta<1$, we conclude that $\sigma_{z r} \sim \tilde{r}^{(\beta-1)\left(2 \alpha_{1}+4 \alpha_{2}-1\right)}$, and in the same way, $\sigma_{z z} \sim$ $\tilde{r}^{(\beta-1)\left(2 \alpha_{1}+4 \alpha_{2}-1\right)}: \sigma_{z r}$ and $\sigma_{z z}$ follow the same power law when $\tilde{r}$ tends to zero. Since the right-hand side of
Eq. (12) is a deltalike charge density, we conclude from Gauss's theorem that the scaling law for $\sigma_{z r}$ and $\sigma_{z z}$ is also $\sigma_{z r} \sim \sigma_{z z} \sim\left(1 / \tilde{r}^{2}\right)$. Therefore, $\tilde{r}^{(\beta-1)\left(2 \alpha_{1}+4 \alpha_{2}-1\right)} \sim \tilde{r}^{-2}$, and

$$
\beta=\frac{2 \alpha_{1}+4 \alpha_{2}-3}{2 \alpha_{1}+4 \alpha_{2}-1} .
$$

Note that $\beta$ is an increasing function of $\left(\alpha_{1}+2 \alpha_{2}\right)$. It is negative for $\alpha_{1}+2 \alpha_{2}<3 / 2$, and it is always smaller than 1 . The displacement at the application point is finite if the exponents are positive, i.e., if $\alpha_{1}+2 \alpha_{2}>3 / 2$. Otherwise, the material cannot withstand a point load. In the following, we assume that $\alpha_{1}+2 \alpha_{2}>3 / 2$, an ansatz that will be legitimized later.

Since $\delta$ is the unique length scale of the deformation, the coordinates in the deformed configuration can be written as $R=\delta f_{0}(\mathbf{r} / \delta)$ and $Z=\delta g_{0}(\mathbf{r} / \delta)$, where $f_{0}$ and $g_{0}$ are two numerical functions depending only on the constitutive law of the elastic medium. The invariants $I_{1}$ and $I_{2}$, and thus the elastic energy density, are dimensionless functions depending only on $\mathbf{r} / \delta$. The elastic energy due to the deformation induced by the point load is

$$
\mathcal{E}_{\mathrm{el0}}=\iiint_{z>0} W_{0} \mathrm{~d}^{3} \mathbf{r}
$$

where $W_{0}$ is the elastic energy density evaluated at $(\tilde{r}, z)$ for the deformation field resulting from the normal point force:

$$
W_{0}(\tilde{r}, z)=W\left(I_{1}\left(\frac{\mathbf{r}}{\delta}\right), I_{2}\left(\frac{\mathbf{r}}{\delta}\right)\right) .
$$

Note that $W$ is assumed to scale as $I_{1}^{\alpha_{1}} I_{2}^{\alpha_{2}}$ just for the strains encountered at $\tilde{r} \ll \delta$. We therefore use a generic expression for the elastic energy density in Eq. (18) in order to take into account the contributions of the strain in the whole material.

The convergence of the integral in Eq. (17) is ensured since the work done by the applied force is finite for $\alpha_{1}+2 \alpha_{2}>\frac{3}{2}$. On the contrary, the integral in Eq. (17) diverges for $\alpha_{1}+2 \alpha_{2}<\frac{3}{2}$.

Since the shear modulus $\mu$ is defined from $W\left(I_{1}, I_{2}\right)$ as [28]

$$
\mu=2\left(\frac{\partial W}{\partial I_{1}}(0,0)+\frac{\partial W}{\partial I_{2}}(0,0)\right),
$$

one introduces $\mathcal{W}_{0}$, the dimensionless elastic energy density, as $\mathcal{W}_{0}=W_{0} / \mu$. It depends on the "shape" of the nonlinearities of the strain energy density function and is independent of the shear modulus $\mu$. Furthermore, as $\mathcal{W}_{0}$ is a function of $\mathbf{r} / \delta$ only, Eq. (17) yields, for $\alpha_{1}+2 \alpha_{2}>\frac{3}{2}$,

$$
\mathcal{E}_{\mathrm{e} 10}=\mu \delta^{3} c_{0}
$$


where $c_{0}$ is a dimensionless parameter that depends on the nonlinear shape of the constitutive equation of the elastic material, but it depends neither on $\delta$ nor on $\mu$ :

$$
c_{0}=\iiint_{z>0} \mathcal{W}_{0}\left(\frac{\mathbf{r}}{\delta}\right) \mathrm{d}^{3}\left(\frac{\mathbf{r}}{\delta}\right)
$$

We stress that Eq. (20) is valid only for $\alpha_{1}+2 \alpha_{2}>\frac{3}{2}$ (such that $c_{0}$ is defined).

\section{Elastic energy for a finite sphere}

In this section, we demonstrate that the elastic energy associated with a normal pointlike force is equal to the elastic energy corresponding to a finite sphere of radius $a$, provided that (i) the downshifts $(\delta)$ are identical in both cases, and (ii) the strain energy density function follows the condition for the convergence of the integral in Eq. (17), a condition which is automatically fulfilled by any real elastic material. We therefore conclude that Eq. (20) gives the elastic energy of an engulfed sphere of any radius $a$, provided that $a \ll \delta$.

We consider a heavy bead of radius $a$ and denote $\delta$ its vertical downshift, which is supposed to be far larger than $a$ [Fig. 4(c)].

(i) Since the unique relevant length scale for $\tilde{r} \gg a$ is $\delta$, the displacement field reduces in this range of $\tilde{r}$ to the displacement field for a point load with the same penetration depth $\delta: Z=\delta g_{0}(\mathbf{r} / \delta)$, where $g_{0}$ has been defined in Sec. III C.

(ii) The power law for $Z-\delta$ with the exponent given by Eq. (16) applies in the intermediate range $a \ll \tilde{r} \ll \delta$. Combining it with the previous expression of $Z$ (valid for $\tilde{r} \gg a$ ) yields

$$
Z-\delta \sim \delta\left(\frac{\tilde{r}}{\delta}\right)^{\beta}=\delta\left(\frac{a}{\delta}\right)^{\beta}\left(\frac{\tilde{r}}{a}\right)^{\beta}
$$

(iii) Close to the bead $(\tilde{r} \ll \delta)$, the unique relevant length scale is $a$. Note that $Z-\delta$ can be expressed as

$$
Z-\delta=K g_{1}\left(\frac{\mathbf{r}}{a}\right)
$$

where $g_{1}$ is a numerical function and the constant $K$ (with the dimension of length) depends on $a$ and also on the far-field deformation, i.e., on $\delta$. Comparing Eq. (22) with the general expressions for $Z$ in the range $\tilde{r} \ll \delta$, one obtains $K=\delta(a / \delta)^{\beta}$.

A similar expression for $R$ can be obtained with the exponent $(3-\beta) / 2$, leading to negligible contributions for the elastic energy.

For $\tilde{r} \ll \delta$, the first invariant $I_{1}$ scales as $Z_{, z}^{2}$ and $Z_{, r}^{2}$, and the second invariant $I_{2}$ scales as $Z_{, z}^{4}$ and $Z_{, r}^{4}$. The (dimensionless) strain energy density function

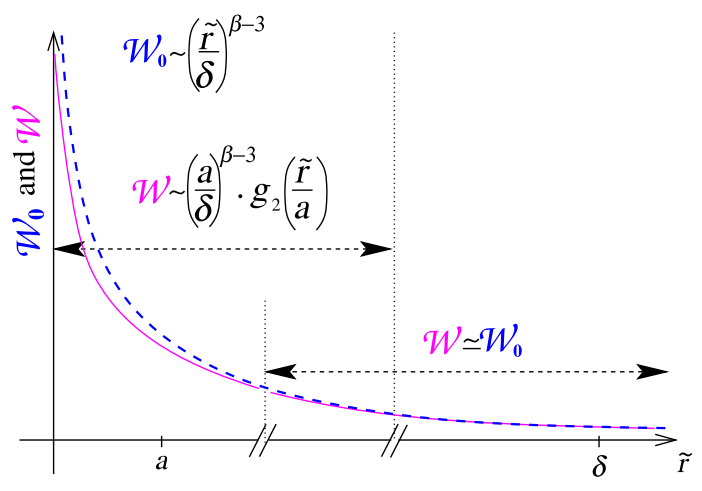

FIG. 5. Sketch of the (dimensionless) energy density profiles $\mathcal{W}_{0}$ (e.g., dotted line) and $\mathcal{W}$ (e.g., solid line) as a function of the distance $\tilde{r}$ to the initial contact point. The dashed horizontal double arrows highlight two domains, corresponding to (i) $\tilde{r} \gg a$, where the (dimensionless) elastic energy densities $\mathcal{W}$ and $\mathcal{W}_{0}$ are similar, and (ii) $\tilde{r} \ll \delta$, where they are different.

$\mathcal{W} \sim I_{1}^{\alpha_{1}} I_{2}^{\alpha_{2}}$ can thus be written for $\tilde{r} \ll \delta$ [using Eq. (23) with the expression of $K]$ as $\mathcal{W} \sim(a / \delta)^{\beta-3} g_{2}(\mathbf{r} / a)$. In the range $\tilde{r} \gg a, \mathcal{W} \simeq \mathcal{W}_{0}$, with $\mathcal{W}_{0}$ being the dimensionless elastic energy density of the point-load problem (Fig. 5).

Moreover, in the range $\tilde{r} \ll \delta$, one finds from the scaling laws of Sec. III C, $\mathcal{W}_{0} \sim(r / \delta)^{\beta-3} \sim(r / a)(a / \delta)^{\beta-3}$.

One concludes that, for any $\tilde{r}, \quad \mathcal{W}-\mathcal{W}_{0} \sim$ $(a / \delta)^{\beta-3} g_{3}(\mathbf{r} / a)$, with $g_{3}$ a numerical function (depending neither on $\delta$ nor on $a$ ) whose limit as its argument approaches $\infty$ equals 0 . Thus, we obtain

$$
\begin{aligned}
& \iiint_{z>0}\left(\mathcal{W}-\mathcal{W}_{0}\right) \mathrm{d}^{3} \mathbf{r} \\
& \quad=\left(\frac{a}{\delta}\right)^{\beta-3} a^{3} \iiint_{z>0} g_{3}\left(\frac{\mathbf{r}}{a}\right) \mathrm{d}^{3}\left(\frac{\mathbf{r}}{a}\right) \sim \delta^{3}\left(\frac{a}{\delta}\right)^{\beta} .
\end{aligned}
$$

Since one assumes again that $\alpha_{1}+2 \alpha_{2}>\frac{3}{2}, \beta>0$, and the difference of the elastic energy with the bead of radius $a$, $\mathcal{E}_{\text {el }}$, to the elastic energy with the point load, $\mathcal{E}_{\text {el0 }}$, is negligible with respect to $\delta^{3}$ [since $(a / \delta)^{\beta} \ll 1$ ]. One concludes from Eq. (20) that the elastic energy $\mathcal{E}_{\text {el }}$ is proportional to $\delta^{3}$.

Note that if $\alpha_{1}+2 \alpha_{2}$ were smaller than $3 / 2$, the nearfield part of the elastic energy would not be negligible anymore compared to the far-field contribution.

\section{E. Strain energy density function}

In Secs. III C and III D, the strain energy density function has been assumed to scale as $I_{1}^{\alpha_{1}} I_{2}^{\alpha_{2}}$ for the strains encountered at $\tilde{r} \ll \delta$. It has been demonstrated that the elastic energy corresponding to the downshift of a sphere of finite radius $a$ over the distance $\delta$ is proportional to $\delta^{3}$ in the limit $\delta \gg a$ if $\alpha_{1}+2 \alpha_{2}>3 / 2$. In this section, we explain why this choice for the strain energy density function does 
not limit the generality of the theory and, therefore, why the theory can be applied for any real elastic material.

It is worth considering the case of an elastic material for which the strain energy density function increases more slowly than $I_{1}^{\alpha_{1}} I_{2}^{\alpha_{2}}$ with $\alpha_{1}+2 \alpha_{2}=3 / 2$. For $\tilde{r} \ll \delta$, we demonstrated that $Z-\delta=\delta(a / \delta)^{\beta} g_{1}(\mathbf{r} / a)$ and then $Z_{, z} \sim(\delta / a)^{1-\beta}$, showing that the strain is arbitrarily large in the limit $\delta \gg a$ if $\beta<1$, i.e., if $\alpha_{1}+2 \alpha_{2}<3 / 2$. However, because of the finite maximum stretch of the polymer chains constituting the material, the maximum stretching of any real elastic rubberlike material is bounded. A divergence of the strain energy function occurs at this maximum stretching. This divergence results in a steeper and steeper increase of $\mathcal{W}$ with $I_{1}, I_{2}$, or both, which is associated with increasing values of the exponents $\alpha_{1}$ and/ or $\alpha_{2}$ [28].

In order to illustrate this last point, let us consider a particular strain energy density function, for instance, the Gent hyperelastic model $[21,30]$. The strain energy density function of this model has a singularity when the first invariant $I_{1}$ reaches a limiting value. It is plotted in Fig. 6: Deviations from the initial neo-Hookean behavior $\left(\alpha_{1}=1\right.$ and $\alpha_{2}=0$ ) yield an increasingly stiffer and stiffer strain energy density function. At any value $I_{10}$ of $I_{1}$, one can define the local exponent $\alpha_{10}=\left(I_{1} / \mathcal{W}\right)\left(\mathrm{d} \mathcal{W} / \mathrm{d} I_{1}\right)$ so that, at the vicinity of $I_{10}$, the strain energy density function is $\mathcal{W} \sim I_{1}^{\alpha_{10}}$ locally. Beyond a certain finite value $I_{10}^{*}$ of $I_{10}$, the exponent is larger than $3 / 2$, ensuring that for any $I_{1}$ larger than $I_{10}^{*}$, the condition $\alpha_{1}+2 \alpha_{2}>3 / 2$ is fulfilled. Note that values of $I_{1}$ larger than $I_{10}^{*}$ are automatically reached; otherwise, the strain would diverge around $\tilde{r} \sim 0$, leading to arbitrarily high values of $I_{1}$ (as explained above).

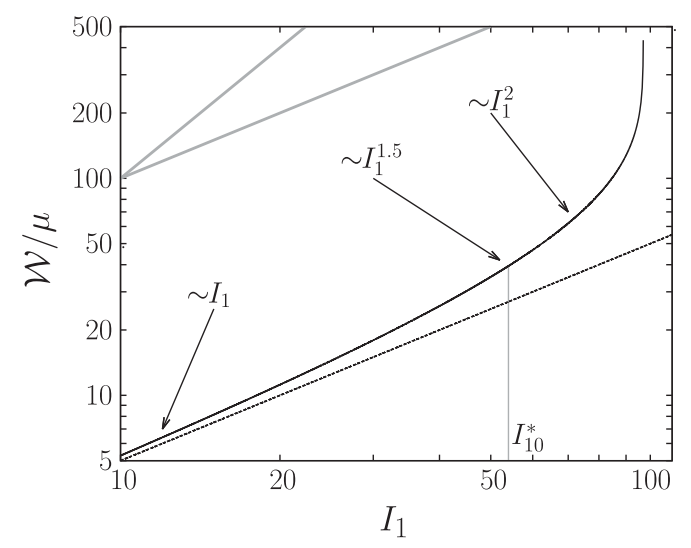

FIG. 6. Strain energy density function given by the incompressible Gent material model $W=-\frac{1}{2} \mu J_{m} \log \left\{1-\left[\left(I_{1}-3\right) / J_{m}\right]\right\}$ [21], with $J_{m}=97$, plotted in $\log -\log$ scales (black solid line). Note that $J_{m}+3$ is the limiting value of the first invariant $I_{1}$. The strain energy density function for the neo-Hookean material is plotted with a dashed line. The two gray straight lines indicate slopes 1 and 2, i.e., values of the local exponent $\alpha_{1}$ equal to 1 and 2. Here, $I_{10}^{*}$ is the value of the first invariant beyond which the local exponent is larger than $3 / 2$.
What is illustrated using the example of the Gent model is general and can be applied to any elastic constitutive law involving $I_{1}$ and $I_{2}$ of a real elastic material: Starting from low to moderate strains for which the neo-Hookean model is expected to apply far from the bead, energy density functions stiffer than $I_{1}^{\alpha_{1}} I_{2}^{\alpha_{2}}$ with $\alpha_{1}+2 \alpha_{2}>\frac{3}{2}$ are necessarily encountered next to the bead for any real elastic material.

A material for which the failure limit is reached before the strain energy density is stiffer than $I_{1}^{\alpha_{1}} I_{2}^{\alpha_{2}}$ with $\alpha_{1}+2 \alpha_{2}>3 / 2$ would not be able to sustain the heavy sphere; thus, it would be drilled by the sphere. These cases can be definitively excluded for the experiments we are dealing with since no fracture, plasticity, or creep have been observed during these experiments.

One concludes that for any elastic material, the elastic energy of the gel is given by Eq. (20) within the limit $\delta \gg a$.

\section{F. Equilibrium condition}

For an engulfed bead of radius $a$ deposited on the initially flat surface of an elastic solid of shear modulus $\mu$ with a density contrast $\Delta \rho$, the gravity energy $\mathcal{E}_{\mathrm{gr}}$ and the elastic energy $\mathcal{E}_{\text {el }}$ have been derived separately for any arbitrary downshift $\delta$ (assuming $\delta \gg a$ ), respectively, in Secs. III A and III D: $\mathcal{E}_{\mathrm{gr}} \simeq \frac{4}{3} \pi a^{3} \Delta \rho g \delta$ and $\mathcal{E}_{\text {el }} \simeq$ $\mathcal{E}_{\text {el0 }}=c_{0} \mu \delta^{3}$. Note that $c_{0}$ is a dimensionless parameter depending neither on $\delta, \mu$, nor $a$. Upon a change of the substrate (e.g., a change in the gel composition), the shear modulus $\mu$ will certainly change. Note that $c_{0}$ will also change if $W / \mu$ changes. In the limit of small deformations, $W / \mu \sim \frac{1}{2} I_{1}$ for any isotropic and incompressible elastic material. The possible change in $c_{0}$ therefore comes from the specific elastic behavior of the substrate at finite deformations.

The total energy, $\mathcal{E}_{\text {el }}+\mathcal{E}_{\text {gr }}$, is at its minimum in the equilibrium state. The $\delta$ derivatives of $\mathcal{E}_{\mathrm{gr}}$ and $\mathcal{E}_{\mathrm{el}}$ are therefore equal, giving the scaling law valid for $\delta \gg a$, for a given elastic material:

$$
\frac{\delta}{\delta_{0}}=\sqrt{\frac{4 \pi}{9 c_{0}}} \cdot\left(\frac{a}{\delta_{0}}\right)^{3 / 2},
$$

with $\delta_{0}=(\mu / \Delta \rho g)$. The crucial point is the nondependence of $c_{0}$ with respect to $a$. Note that $c_{0}$ depends only on the nonlinear elastic properties of the elastic solid, and therefore, for a given elastic material, the downshift of the bead, $\delta$, is expected from theory to be proportional to the radius of the bead to the power $\frac{3}{2}$ times the square root of $\Delta \rho g$.

Note that we have assumed the beads are spherical, although this is not a crucial point in this theory: If it is 
compact with all typical lengths of the same order $a$, the previous scaling arguments apply.

\section{DISCUSSION AND CONCLUSION}

The theoretical analysis presented above provides an understanding of the experimentally observed scaling law. It arises from a specific property of the system: The elastic energy does not depend on the radius of the sphere.

The scaling behavior found for the downshift of spheres with different radii and/or different densities is general, and the specific property of the elastic material has an effect only on the coefficient $c_{0}$ in this law [Eq. (25)]. These predictions are in quantitative agreement with the observations: An experimental scaling law for the depth is a function of the radius raised to an exponent close to $3 / 2$, and the prefactor is not only related to the linear elastic properties $(\mu)$ but also to the nonlinear features of the elastic material. These nonlinear features being a priori distinct from one gel to another explains why plotting $\delta / \delta_{0}$ as a function of $a / \delta_{0}$ for different gel compositions yields slightly different curves for the extremely soft gels but more or less a master curve for the stiffer gels. Thus, the prefactor in the scaling law depends not only on the shear modulus of the solid but also on the elastic behavior at large deformations. This provides a way to assess some characteristics such as strain stiffening properties of elastic materials under large strains.

The range of applications of the derived scaling goes far beyond the description of the elastobuoyancy phenomenon. For instance, we show here that these soft gels can sustain much larger deformations than those induced by the elastobuoyant spheres when a sharp-pointed needle is indented into the gel, through a layer of silicone oil to minimize friction, without fracturing the gel [Fig. 7(a)]. The fact that no fracture occurs in the gel is evident with a dyed interface that returns to its original state following the removal of the needle from inside the gel. In this case, the indentation force $(P)$ varies as the square of the displacement $(\delta)$ [Fig. 7(b)], a scaling that was reported previously with studies involving large deformations of soft solids [15] but not with a sharply pointed load. In this current study, we also paid special attention to the removal of lateral solidlike friction, which can contribute to the indentation load through complex coupling between the normal stress difference and the shear strain in a nonlinear solid [31]. The scaling for the energy for the case of large deformations can be directly checked from these observations: $P \sim \partial \mathcal{E}_{\mathrm{el}} / \partial \delta \sim \delta^{2}$, i.e., $\mathcal{E}_{\mathrm{el}} \sim \delta^{3}$. This scaling for the force is in unison with the exponent $3 / 2$ for the penetration depth of an elastobuoyant sphere in the limit of the large deformations. It is observed here within a wider range of strains, an indication that the scaling law of elastobuoyant spheres in these gels is valid for larger and heavier beads, and/or with even softer gels. Furthermore, this testifies that our analysis (a)

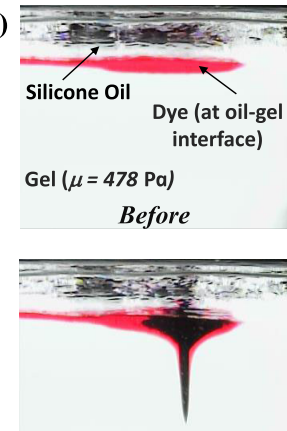

Loaded with a sharp point

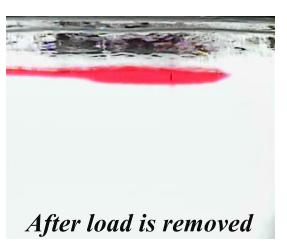

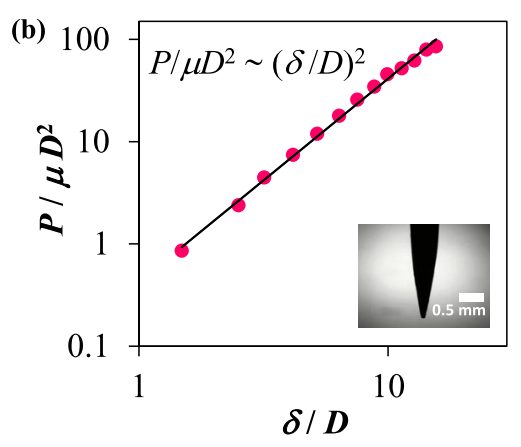

(c)

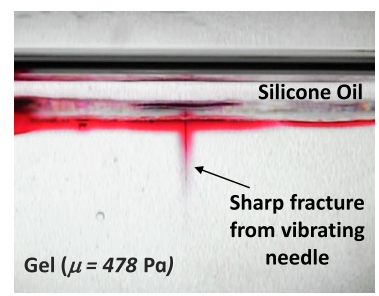

FIG. 7. (a) Snapshots of the experiment of loading a needle (diameter $D$ and grafted with about 5-nm layer of polydimethylsiloxane chains) inside the gel with a thin layer of silicone oil (AR20, Sigma Aldrich) on its surface. The oil above the gel reduces friction by lubricating the contact between the needle and the gel during indentation. The red dye demarcates the interface between the gel and silicone oil. After unloading the needle from the gel, the dyed interface retracts to its original position, showing that there was no fracture in the gel. (b) For these experiments of loading a sharp needle (inset) inside the gel $(\mu=478 \mathrm{~Pa})$, the nondimensional force $P / \mu D^{2}$ varies almost as the square of the nondimensional displacement $\delta / D$. An uncertainty analysis yields the value of the power of $\delta / D$ as $1.99 \pm 0.03$. (c) When the same needle is indented in the gel while undergoing vertical vibrations, a fine fracture is induced by the indentation. The needle undergoes square-wave oscillations (amplitude of vibration of about $0.64 \mathrm{~mm}$ ) along its axial direction, which were generated by a waveform generator (Agilent, Model 33120A), connected to a mechanical oscillator (Pasco Scientific, Model No: SF-9324) via an amplifier (Sherwood, Model No: RX-4105).

is general and goes beyond the phenomenon of elastobuoyancy.

While on one hand such materials could be potentially used for various applications where one would need to make use of large deformations, on the other hand, one could also ask the question the following question: How could one induce fracture $[15,32]$ in such systems that are otherwise capable of handling such huge strains without failure? The gels we use in the experiments are stiff in a sense because they are made of polymers that cannot stretch beyond a given limit; thus, the question of the initiation of fracture in such systems arises. In the scenario where a sharp slender body has hugely deformed the gel, such that the elastic energy is distributed over a large volume $\left(\sim \delta^{3}\right)$ as explained by the theory, there is not enough energy available locally to initiate fracture. 
This applies regardless of the shape of the body, provided that its characteristic lengths are far smaller than the static penetration depth $\delta$. Nevertheless, when the same sharppointed object undergoes vertical vibrations while penetrating the gel, through the silicone oil to minimize lateral friction which could potentially lead to irregular fracture, it induces a sharp cut in an irreversible way [Fig. 7(c)] much more easily. This is the case even when the lateral friction-induced interfacial jamming between the gel and the needle is eliminated. We hypothesize that this sharp cut is facilitated by the local rheological stiffening of the gel, which is made possible by not allowing sufficient time for it to relax from a highfrequency-high-modulus state to a relaxed one. Such vibration-assisted puncturing of soft materials was demonstrated with marked precision as early as almost 50 years ago, even with a single cell [33]; however, the detailed mechanics of such kinds of fracture are not well known. Puncturing of such soft gels assisted with vibration thus provides a good model system to study such an important phenomenon, which has immense practical applications, including precision surgery on soft tissues in humans. The analysis presented in this paper provides the motivation required for developing such a model, in which the local fields are manipulated by the frequency response of the gel, while an overarching large deformation field surrounds the locality.

In this paper, we have shown that the depth of a bead scaling as exponent $3 / 2$ for the radius is independent of the strain-stress relation, provided that the increase in the elastic energy density $(W)$ with the strain is stiff enough.

The generality of the scaling and the observations are supported by indentation experiments using a sharppointed needle that follows a similar trend for large deformations as that observed in the elastobuoyancy phenomenon. Generic behaviors for elastic materials undergoing large and complex deformations can therefore be identified, going beyond scaling arguments, which are blind to the crucial effect of strain stiffening. This work sheds new light on the mechanics of extremely large elastic deformations that are crucial in various emerging techniques that even encompass an important procedure such as the computer-assisted surgery involving human organs [34-36], which are indeed nonlinear materials that undergo large deformations. In all these important fields, an ideal neo-Hookean model is used that, according to our current work, is not a suitable choice for such studies. The danger is that one may miss the divergences of the solution that is inherent in the ideal neo-Hookean model because the full solution is not used. The work presented here provides a redress to the possible pitfalls that one may embark upon in studying extreme deformations of nonlinear materials, thereby also offering possible benchmarks for numerical simulations and even opportunities to formulate new simulation methods.

\section{ACKNOWLEDGMENTS}

S. M. thanks the French National Research Agency for its support (FAPRES, Contract No. ANR-14-CE32-0005).

[1] P. G. de Gennes, Wetting: Statics and Dynamics, Rev. Mod. Phys. 57, 827 (1985).

[2] Y. Pomeau, Recent Progress in the Moving Contact Line Problem: A Review, Comp. Rend. Mécanique 330, 207 (2002).

[3] K. L. Johnson, Contact Mechanics, Cambridge Books Online (Cambridge University Press, Cambridge, England, 1985).

[4] J. Kim, J. Yoon, and R. C. Hayward, Dynamic Display of Biomolecular Patterns through an Elastic Creasing Instability of Stimuli-Responsive Hydrogels, Nat. Mater. 9, 159 (2010).

[5] S. Mora, T. Phou, J. M. Fromental, L. M. Pismen, and Y. Pomeau, Capillarity Driven Instability of a Soft Solid, Phys. Rev. Lett. 105, 214301 (2010).

[6] S. Mora, M. Abkarian, H. Tabuteau, and Y. Pomeau, Surface Instability of Soft Solids under Strain, Soft Matter 7, 10612 (2011).

[7] R. W. Style, C. Hyland, R. Boltyanskiy, J. S. Wettlaufer, and E. R. Dufresne, Surface Tension and Contact with Soft Elastic Solids, Nat. Commun. 4, 2728 (2013).

[8] B. Saintyves, O. Dauchot, and E. Bouchaud, Bulk Elastic Fingering Instability in Hele-Shaw Cells, Phys. Rev. Lett. 111, 047801 (2013).

[9] S. Mora, C. Maurini, T. Phou, J. M. Fromental, B. Audoly, and Y. Pomeau, Solid Drops: Large Capillary Deformations of Immersed Elastic Rods, Phys. Rev. Lett. 111, 114301 (2013).

[10] A. Jagota, D. Paretkar, and A. Ghatak, Surface-TensionInduced Flattening of a Nearly Plane Elastic Solid, Phys. Rev. E 85, 051602 (2012).

[11] M. K. Chaudhury, A. Chakrabarti, and A. Ghatak, Adhesion-Induced Instabilities and Pattern Formation in Thin Films of Elastomers and Gels, Eur. Phys. J. E 38, 82 (2015).

[12] T. Tallinen, J. Y. Chung, J. S. Biggins, and L. Mahadevan, Gyrification from Constrained Cortical Expansion, Proc. Natl. Acad. Sci. U.S.A. 111, 12667 (2014).

[13] X. Zhao and Z. Suo, Theory of Dielectric Elastomers Capable of Giant Deformation of Actuation, Phys. Rev. Lett. 104, 178302 (2010).

[14] A. Chakrabarti and M. K. Chaudhury, Direct Measurement of the Surface Tension of a Soft Elastic Hydrogel: Exploration of Elasto-Capillary Instability in Adhesion, Langmuir 29, 6926 (2013).

[15] S. Fakhouri, S. B. Hutchens, and A. J. Crosby, Puncture Mechanics of Soft Solids, Soft Matter 11, 4723 (2015).

[16] G. G. Stokes, On the Theories of the Internal Friction of Fluids in Motion and of the Equilibrium and Motion of Elastic Solids, Trans. Cambridge Philos. Soc. 8, 287 (1845).

[17] Lord Rayleigh, Theory of Sound (Dover, New York, 1922), Vol. II. 
[18] R. S. Rivlin, Large Elastic Deformations of Isotropic Materials. IV. Further Developments of the General Theory, Phil. Trans. R. Soc. A 241, 379 (1948).

[19] R. W. Ogden, Large Deformation Isotropic Elasticity-On the Correlation of Theory and Experiment for Incompressible Rubberlike Solids, Proc. R. Soc. A 326, 565 (1972).

[20] O. H. Yeoh, Some Forms of the Strain Energy Function for Rubber, Rubber Chem. Technol. 66, 754 (1993).

[21] A. N. Gent, A New Constitutive Relation for Rubber, Rubber Chem. Technol. 69, 59 (1996).

[22] R. Mangan, M. Destrade, and G. Saccomandi, Strain Energy Function for Isotropic Non-linear Elastic Incompressible Solids with Linear Finite Strain Response in Shear and Torsion, Extreme Mech. Lett. 9, 204 (2016).

[23] A. Chakrabarti and M. K. Chaudhury, Elastocapillary Interaction of Particles on the Surfaces of Ultrasoft Gels: A Novel Route to Study Self-Assembly and Soft Lubrication, Langmuir 30, 4684 (2014).

[24] D. S. Rimai, D. J. Quesnel, and A. A. Busnaina, The Adhesion of Dry Particles in the Nanometer to MicrometerSize Range, Colloids Surf. A 165, 3 (2000).

[25] S. Mondal, M. Phukan, and A. Ghatak, Estimation of Solid Liquid Interfacial Tension Using Curved Surface of a Soft Solid, Proc. Natl. Acad. Sci. U.S.A. 112, 12563 (2015).

[26] See Supplemental Material at http://link.aps.org/supplemental/ 10.1103/PhysRevX.6.041066 for experimental check of the reversibility of the deformation in the gels by imposing an additional magnetic force to a steel bead.

[27] D. Vella, Floating Versus Sinking, Annu. Rev. Fluid Mech. 47, 115 (2015).

[28] R. W. Ogden, Non-Linear Elastic Deformations (Ellis Horwood Limited, Chichester, 1984).

[29] An Introduction to the Calculus of Variations, edited by C. Fox (Dover, New York, 1987).

[30] A. N. Gent, Elastic Instabilities in Rubber, Int. J. Nonlinear Mech. 40, 165 (2005).

[31] M. K. Chaudhury, Viewpoint: A Cut above the Rest, Physics 5, 139 (2012).

[32] S. Das and A. Ghatak, Puncturing of Soft Gels with MultiTip Needles, J. Mater. Sci. 46, 2895 (2011).

[33] T. K. Chowdhury and F. M. Snell, A Microelectrode Study of Electrical Potentials in Frog Skin and Toad Bladder, Biochim. Biophys. Acta 94, 461 (1965).

[34] S. Cotin, H. Delingette, and N. Ayache, Real-Time Elastic Deformations of Soft Tissues for Surgery Simulation, IEEE Trans. Visual. Comput. Graph. 5, 62 (1999).

[35] A. Liu, F. Tendick, K. Cleary, and C. Kaufmann, A Survey of Surgical Simulation: Applications, Technology, and Education, Presence-Teleop. Virt. 12, 599 (2004).

[36] Z. A. Taylor, M. Cheng, and S. Ourselin, High-Speed Nonlinear Finite Element Analysis for Surgical Simulation Using Graphics Processing Units, IEEE Trans. Med. Imaging 27, 650 (2008). 